\title{
Clinical and Molecular Update on the Fourth Reported Family with Hamamy Syndrome
}

\author{
André Mégarbanéa ${ }^{a, b}$ Sayeeda Hana ${ }^{c}$ Hala Mégarbanéd $^{d}$
}

Christel Castro $^{e}$ Sylvain Baulande ${ }^{f}$ Audrey Criqui ${ }^{f}$ Nathalie Roëckel-

Trevisiol $^{\mathrm{e}}$ Christel Dagher ${ }^{\mathrm{a}}$ Mahmoud Taleb Al-Ali ${ }^{\mathrm{c}}$ Jean-Pierre Desvignes ${ }^{\mathrm{e}}$

Daniel Mahfoud ${ }^{g}$ Stephany El-Hayek ${ }^{c}$ Valérie Delague ${ }^{\mathrm{e}}$

${ }^{a}$ Department of Human Genetics, Gilbert and Rose-Marie Ghagoury School of Medicine, Lebanese American University, Byblos, Lebanon; ' Institut Jérôme Lejeune, Paris, France; 'Centre for Arab Genomic Studies, Dubai, UAE; ${ }^{\mathrm{d} D e p a r t m e n t}$ of Dermatology, Balamand University, Saint George Hospital, Beirut, Lebanon; ${ }^{\mathrm{e} A i x}$ Marseille Univ, Inserm, MMG, U 1251, Marseille, France; fGénopole Campus 2, PartnerChip, Evry, France; 9Department of Radiology, Gilbert and Rose-Marie Ghagoury School of Medicine, Lebanese American University, Byblos, Lebanon

\section{Keywords}

Dysmorphology · Exome sequencing · Hamamy · IRX5

\begin{abstract}
We report on 2 cousins, a girl and a boy, born to first-cousin Lebanese parents with Hamamy syndrome, exhibiting developmental delay, intellectual disability, severe telecanthus, abnormal ears, dentinogenesis imperfecta, and bone fragility. Whole-exome sequencing studies performed on the 2 affected individuals and one obligate carrier revealed the presence of a homozygous c.503G >A (p.Arg168His) missense mutation in IRX5 in both sibs, not reported in any other family. Review of the literature and differential diagnoses are discussed.

(c) 2021 S. Karger AG, Basel
\end{abstract}

\section{Introduction}

Neural crest cells (NCCs), a transitory population of multipotent stem cells that arise in the embryonic ectoderm germ layer, are responsible for the development of a variety of tissues, ranging from neurons, glia and mela- nocytes to craniofacial bone, cartilage and connective tissue [Graham, 2003; Watt and Trainor, 2014]. Defects in the migration or differentiation of NCCs result in a broad spectrum of congenital malformations collectively known as neurocristopathies [Vega-Lopez et al., 2018]. Bonnard et al. [2012] studied the functional effect of mutations in IRX5, which modulates the migration of NCCs by repressing the chemokine SDF1, and found it to be associated with Hamamy syndrome.

Hamamy syndrome, initially described in 2 Jordanian siblings by Hamamy et al. [2007], is a rare autosomal recessive congenital malformation affecting the development of the face, brain, blood, heart, bones, and gonads. The Jordanian brothers exhibited striking telecanthus, midface prominence, brachycephaly, and abnormal ears, resulting in a distinct appearance. Intellectual disability, myopia, sensorineural deafness, cryptorchidism, osteopenia, and recurrent fractures were also noted. Since then, only 3 other families have been reported with this condition (Table 1).

Here, we report a multiply consanguineous Lebanese family where 2 cousins presented with Hamamy syndrome. Review of the literature and differential diagnoses are discussed.
Correspondence to:

André Mégarbané, andre.megarbane@lau.edu.lb 


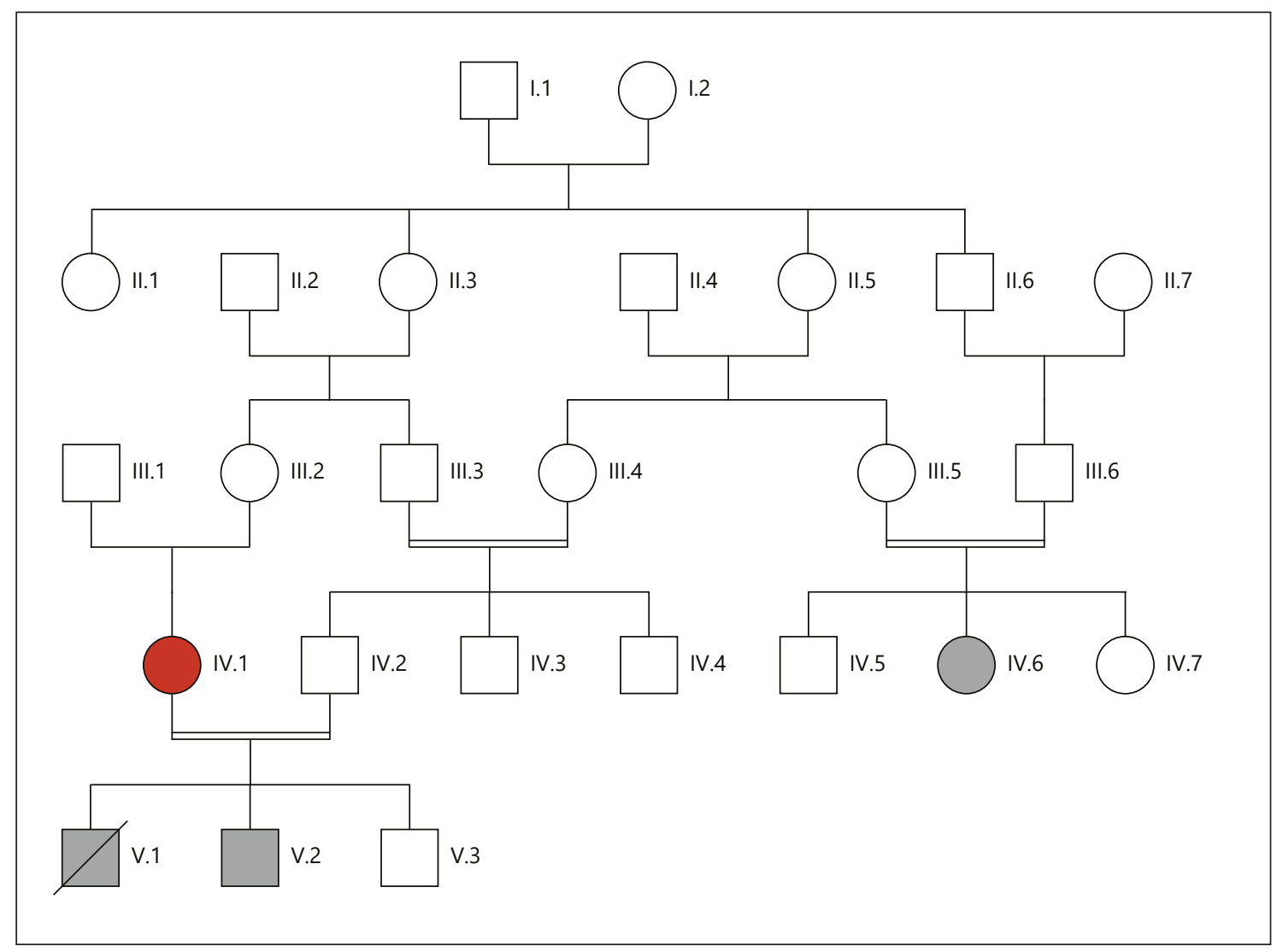

Fig. 1. Pedigree of the studied family. Affected individuals are shaded in black. Whole-exome sequencing was performed on the individual shown in red as well as the 2 affected individuals.

\section{Materials and Methods}

\section{Patients}

The patients, a girl and a boy, are cousins from a multiply consanguineous Lebanese family (Fig. 1). Each underwent a work-up including a thorough clinical evaluation, total body X-rays, echocardiography, karyotyping, and routine blood tests (complete blood count, serum electrolytes, blood glucose levels, cholesterol, thyroid, liver and renal function tests, and phosphatase alkaline levels).

\section{Molecular Analysis}

DNA Isolation

Genomic DNA was isolated from patient blood samples using standard techniques.

Genome-Wide Microarray CGH Analysis

Genomic DNA from the affected boy was amplified and purified. Following denaturation of probe, DNA, hybridization was carried out with the Affymetrix Cytogenetics Whole-Genome 2.7 M Array following the manufacturers' standard protocol. Data was collected using GeneChip ${ }^{\circledR}$ Scanner 30007 G, and CEL files were analyzed using Affymetrix Chromosome Analysis Suite software (ChAS v.1.0.1).

New IRX5 Mutation in Hamamy Syndrome
Whole-Exome Sequencing

Whole-exome sequencing (WES) was carried out on 3 individuals: the 2 affected individuals (IV.6 and V.2) and the unaffected mother IV.1 (Fig. 1). Library preparation, capture, and sequencing were performed on a private sequencing platform (Integragen Genomics, Evry, France). Briefly, exomes were captured and enriched using the in solution Agilent SureSelect Human All Exon kit v3.0 [Sulonen et al., 2011] and then sequenced on an Illumina HiSeq2000, using a paired-end 100-bp read sequencing protocol. Image analysis and base calling were performed using the Illumina Data Analysis Pipeline Software 1.5 with default parameters. Raw data were mapped to the current built of the human genome (hg19) by using BWA 0.75 [Li and Durbin, 2009]. Variant calling was subsequently performed using GATK 2.5.2 [McKenna et al., 2010], and annotation was done with ANNOVAR [Wang et al., 2010].

Variant sorting and filtering was performed using VarAFT (Variant Annotation and Filter tool), a variant filtering and prioritization tool allowing easy and fast selection and segregation of variants from exome or genome data [Desvignes et al., 2018]. Considering autosomal recessive inheritance and the presence of consanguinity in the family, we have segregated the variants from exome data following a homozygous by descent model. More pre- 
Fig. 2. Images highlighting the facial features of patient 1 (a) and patient 2 (b), along with computed tomography $3 \mathrm{D}$ reconstructions of patient $2(\mathbf{c}, \mathbf{d})$.

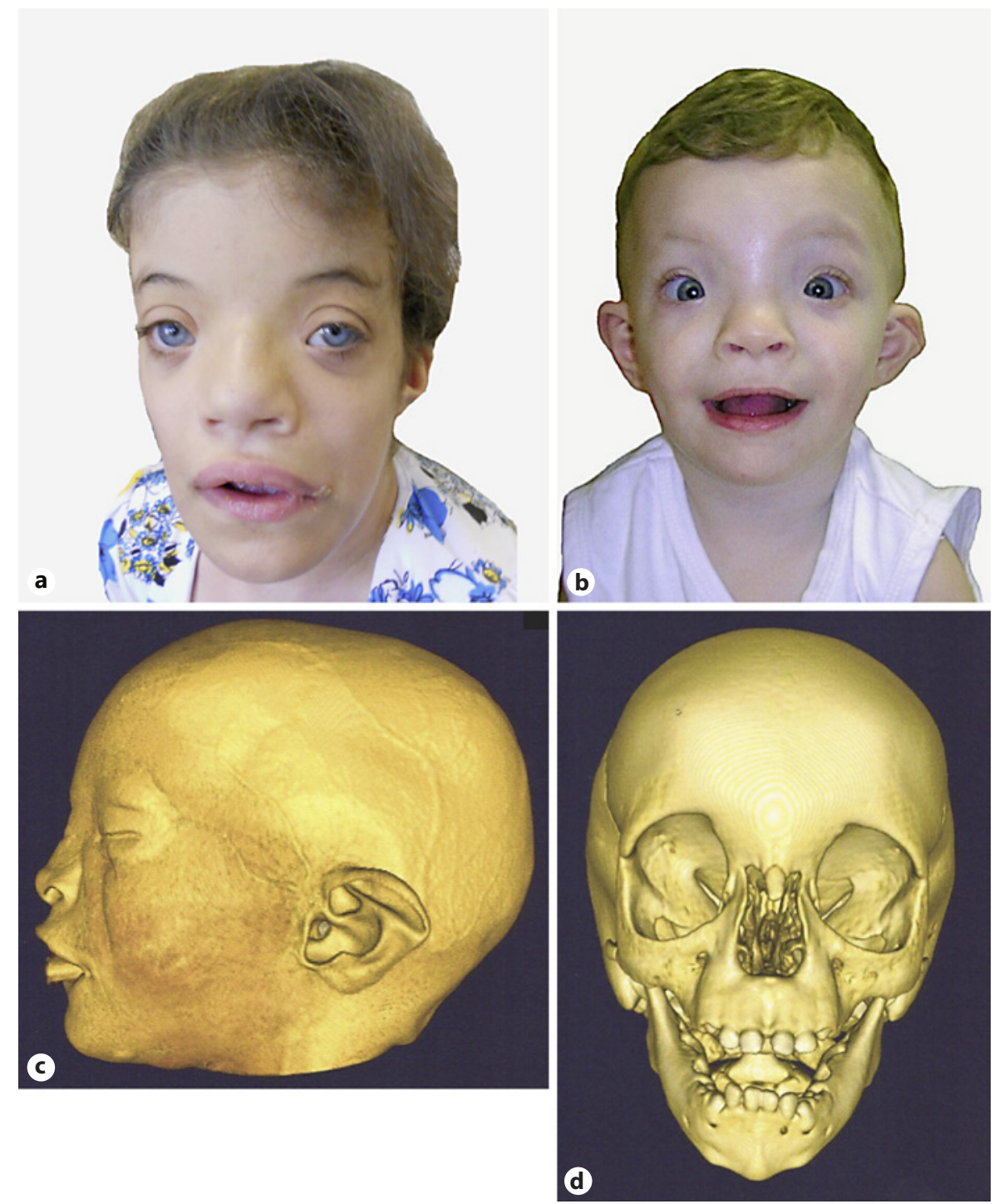

cisely, we have selected homozygous variants shared by the 2 patients (IV.6 and V.2), which were heterozygous in the obligate carrier mother (IV.1). In order to refine the list of candidate nucleotide variations, filtering was performed by removing all variants with allele frequencies $>1 \%$ in the gnomAD database (https://gnomad.broadinstitute.org/). Additional filtering was also performed using our in-house exome database which includes all homozygous variants detected by exome sequencing of 14 Lebanese unaffected individuals.

In order to predict the deleterious effect of the identified sequence variations, different bioinformatics tools were applied: MutationTaster [Schwarz et al., 2014], SIFT [Kumar et al., 2009], PolyPhen-2 [Adzhubei et al., 2010], UMDpredictor [Salgado et al., 2016], and CADD [Rentzsch et al., 2019].

\section{Results}

\section{Clinical Report}

\section{Patient 1}

Patient 1 (IV.6, Fig. 1), a girl, was born at term. Her birth weight was 3,000 g (30th centile), and length $51 \mathrm{~cm}$ (83rd centile). Dysmorphic facial features consisting of a flat forehead, sparse hair and eyebrows, and widely spaced eyes were noted at birth, according to her medical history. From the age of 3 months, recurrent fractures of the femurs started occurring after minor trauma, for which she was operated. Her developmental milestones were delayed, as she walked at 2 and started to say a few words at around the age of 5 years. She was also diag- 
Fig. 3. Images displaying the dental features of patient $1(\mathbf{a})$, a panoramic X-ray of patient 1 (b), and the dental features of patient 2 (c).

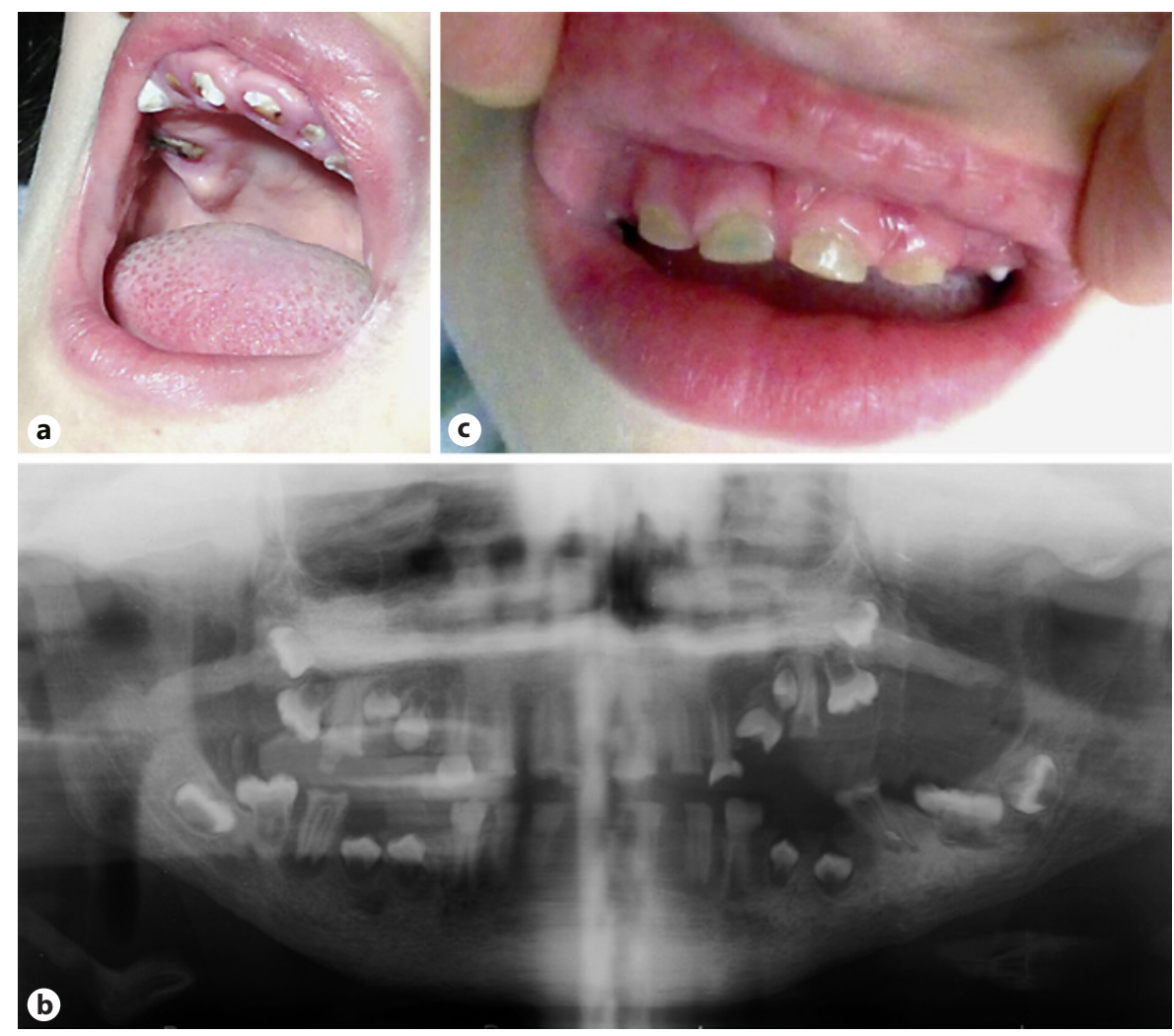

nosed with a diminished audition that was not further investigated.

We saw her when she was 12 years old. She had severe intellectual disability. Her height was $143 \mathrm{~cm}$ (15th centile), weight $44 \mathrm{~kg}$ (66th centile), and her head circumference (OFC) was $56 \mathrm{~cm}$ (95th centile). She had a flat forehead, sparse eyebrows and eyelashes, a broad nasal bridge, a telecanthus with palpebral fissures of $2.8 \mathrm{~cm}$ (Z-score $=-1.28)$, inner canthal distance of $4.5 \mathrm{~cm}(\mathrm{Z}$-score $=1.88)$, outer canthal distance of $10.1 \mathrm{~cm}(Z$-score $=0)$, an interpupillary distance of $7 \mathrm{~cm}(\mathrm{Z}$-score $=1.88)$, dysplastic, low-set ears, flat philtrum, thick lips, and a large mouth (Fig. 2a). Clinical oral examination revealed that all teeth in both dentitions were affected with generalized opalescent tooth discoloration (yellow-brown translucent), enamel frequently separates easily from dentin and wear of enamel that was flushed with the level of the gingiva (Fig. 3a). Radiographic examination based on a panoramic X-ray (Fig. 3b) showed bulbous crowns with cervical constriction, long tapered roots, and progressive obliteration of the pulp chamber.

External genitalia, ophthalmological, neurological and heart examinations, as well as abdominal and heart ultrasound findings and electrocardiogram results, were unremarkable.

New IRX5 Mutation in Hamamy Syndrome

\section{Patient 2}

Patient 2 (V.2, Fig. 1), a boy, was born at term by a Csection after a normal pregnancy. Birth weight was 3,800 $\mathrm{g}$ (81st centile), length $52 \mathrm{~cm}$ (86th centile), and OFC was $37 \mathrm{~cm}$ (97th centile). Severe telecanthus was noted at birth and a spontaneous fracture of the left femur diagnosed at one week.

When he was brought to us for consultation, he was 45 days old. His length was $57 \mathrm{~cm}$ (57th centile), weight $5,100 \mathrm{~g}$ (55th centile), and his OFC $39 \mathrm{~cm}$ (70th centile). His facial features included a flat receding forehead, widely spaced eyes, short palpebral fissures, nearly absent eyebrows, low-set ears, an abnormal left ear, lateral placement of the inner canthus, and cryptorchid testes. His skin was normal, not exhibiting any scars or hyperelasticity (Fig. 2b). Neurological examination, audiometry, and echocardiography were normal. Total body X-rays showed nothing abnormal except for a bent left femur with the presence of a fracture line.

He was able to sit unassisted by the age of 8 months and began walking at around 15 months. Subsequently, between the ages of 15 months and 2 years, he had 3 episodes of left femur fracture that were treated the first 2 times by a cast and the third time by surgery. During this 
operation, a bone biopsy was performed and results of the histology showed the presence of dystrophic remodeling of the bones which suggested a diagnosis of osteogenesis imperfecta. Bilateral dysfunction of the nasolacrimal structures was also noted and treated by nasolacrimal probing twice, but without success.

Computed tomography images performed at age 18 months displayed craniofacial disproportion and dysmorphic appearance of the ear lobes, but did not find a significant increase in the distance between the medial orbital walls. Axial cuts showed a mega cisterna magna with normal appearance of the globes and orbital content (Fig. 2c, d).

By the age of 2, his developmental delay had become more evident. His height was $97 \mathrm{~cm}$ (99th centile), weight $12 \mathrm{~kg}$ (45th centile), and his OFC was $51 \mathrm{~cm}$ (97th centile). He could say a few words and understand simple orders. He had a bilateral inguinal hernia for which he had been operated. The general aspect was unchanged.

Telecanthus was striking with palpebral fissures of 2.6 $\mathrm{cm}(\mathrm{Z}$-score $=1.65)$, inner canthal distance of $4.3 \mathrm{~cm}(\mathrm{Z}$ score $=1.88)$, and outer canthal distance of $9.9 \mathrm{~cm}(\mathrm{Z}$ score $=1.88)$. His hair, eyelashes and eyebrows were sparse; the nasal bridge broad; the philtrum flat; the upper lip thin; the lower lip everted, and the mouth large. There was a generalized opalescent tooth discoloration (bluegray translucent). Teeth were also weaker than normal, making them prone to rapid wear, breakage, and loss, with abrasion and some coronal fracture (Fig. 3c). Radiographic examination based on an occlusal X-ray showed large pulp chambers in the primary dentition. The clinical and radiographic findings sustained a diagnosis of dentinogenesis imperfecta. Shoulders were narrow and sloping. Ophthalmological examination showed the presence of astigmatism. Heart ultrasound and electrocardiogram tests were normal.

A few months later, he suffered a hairline fracture in right femur. X-ray showed bone bowing in the right leg. A second bone biopsy from the crest and fractured area showed no evidence of osteogenesis imperfecta.

Clinical examination of the parents was normal except that the father of patient 2 had a mild telecanthus with palpebral fissures of $3.5 \mathrm{~cm}$ and inner canthal distance of $3.8 \mathrm{~cm}$.

\section{Laboratory Studies}

Results of complete blood counts, serum electrolytes, blood glucose levels, triglycerides, cholesterol, amino acid studies of plasma and urine, urinary screening for organic acids, urinalysis, thyroid, liver (alkaline phosphatase, alanine transaminase, aspartate aminotransferase, and gamma-glutamyl transpeptidase) and renal function (urea, creatinine and albumin), CPK, Ca, P, were all unremarkable in both cousins. Alkaline phosphatase was slightly elevated in patient 2 . Lymphocytic chromosome study with high resolution in R-banding showed a normal 46,XX karyotype for patient 1 (IV.6), and a normal 46,XY for patient 2 (V.2).

\section{Molecular Studies}

Array-CGH analysis for submicroscopic deletions/duplications performed in patient 2 revealed no causal CNVs.

Considering autosomal recessive transmission and the fact that the patients are issued from consanguineous marriages, we analyzed the WES data, assuming identity by descent in the affected individuals. More precisely, we selected variants, which were homozygous in both affected individuals (IV.6 and V.2), and heterozygous in the obligate carrier mother (IV.1). By using this strategy, we first identified 873 variants, of which 120 had a frequency $>1 \%$ in gnomAD. Further filtering on a local Lebanese database allowed to narrow the number of candidate variants to 78. By focusing on variants located in exonic or splice-site regions, we finally ended up with a list of 4 new or excessively rare missense variants: c.503G $>A$ (p.Arg168His) in IRX5 exon 2 (NM_005853), c.1832C > G (p.Thr611Ser) in RFWD3 exon 11 (NM_018124), c.1390G $>$ A (p.Gly464Arg) in SLC27A3 exon 5 (NM_024330), and c.560A $>$ G (p.His187Arg) in ECM1 exon 6 (NM_004425). Interestingly, the mutated genes are located in 2 different homozygous by descent regions: IRX5 and RFWD3 at chromosome 16q12q23, and ECM1 and SLC27A3 at chromosome 1q21.3. In each region, 2 rare and new variants are in linkage disequilibrium.

The variants identified in ECM1 were eliminated by segregation studies. The remaining variants in RFWD3 and SLC27A3 were not considered causal as mutations in the former are associated with Fanconi Anemia, which do not match our patients' phenotype, while $S L C 27 A 3$ is not associated with any disorder. The fourth mutation, c.503G $>$ A (p.Arg168His) in IRX5, (Fig. 4a), was not found in dbSNP or the genome Aggregation Database (gnomAD). Analysis of the variant using pathogenicity prediction tools suggested a damaging effect; it was predicted to be "disease causing" by MutationTaster (score = 29 ) and "deleterious" by CADD (score = 33). Furthermore, the arginine residue affected by this variant is extremely well conserved among species and among the IRX protein family (Fig. 4b). Most importantly, as $2 \mathrm{mu}$ - 


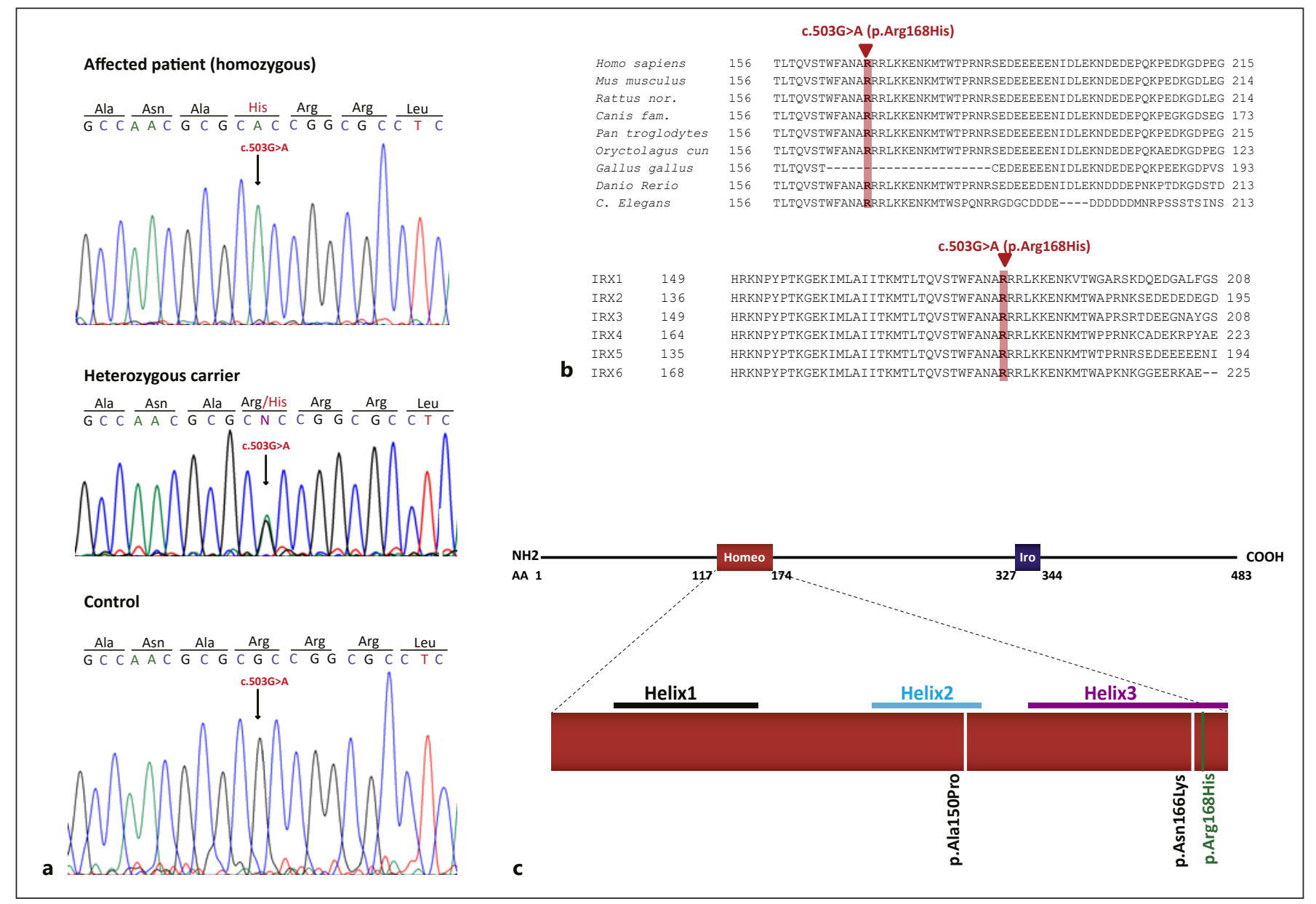

Fig. 4. Mutation identification. a Chromatograms of one patient, the heterozygous mother and a control showing the homozygous c. $503 \mathrm{G}>\mathrm{A}$ (p.Arg168His) mutation identified in exon 2 of IRX5 (NM_005853). b Alignment of IRX5 amino acid sequences between different species (top) and between all members of the Iroquois protein family using CLUSTAL 2.1. The Arginine at position 168 , mutated in our patients, is highlighted in red. It is highly conserved across vertebrates and in the different members of the Iro- quois family. c Schematic representation of the 483 amino acid IRX5 protein (NP_005844) showing the 2 conserved regions: a homeodomain (Homeo) and an Iro box (Ibo). We show the position of the 3 mutations described to date in IRX5 (including ours) in the homeodomain. The 2 mutations previously reported by Bonnard et al. [2012] are indicated in black, while the new variant that we describe in this study is indicated in green. tations in IRX5 had been previously described in Hamamy syndrome (MIM 611174), the p.(Arg168His) IRX5 missense variant was considered the causative mutation in the 2 patients described here.

\section{Discussion}

We report here on 2 patients with Hamamy syndrome from a multiply consanguineous Lebanese family exhibiting developmental delay, intellectual disability, severe telecanthus, abnormal ears, dentinogenesis imperfecta, and bone fragility. This syndrome has so far been identified in 4 other families (Table 1) [Hamamy et al., 2007; Bonnard et al., 2012; Roukoz et al., 2019; Duman et al., 2020].

One striking feature is the severe telecanthus. The latter is a rather frequently reported feature in several dysmorphological syndromes [Dollfus and Verloes, 2004]. These include different chromosomal syndromes and also mendelian syndromes such as frontonasal dysplasia (OMIM 136760), craniofrontonasal syndrome (OMIM 304110), Waardenburg syndrome (193500), Branchiooculofacial syndrome (OMIM 113620), Nablus mask-like 


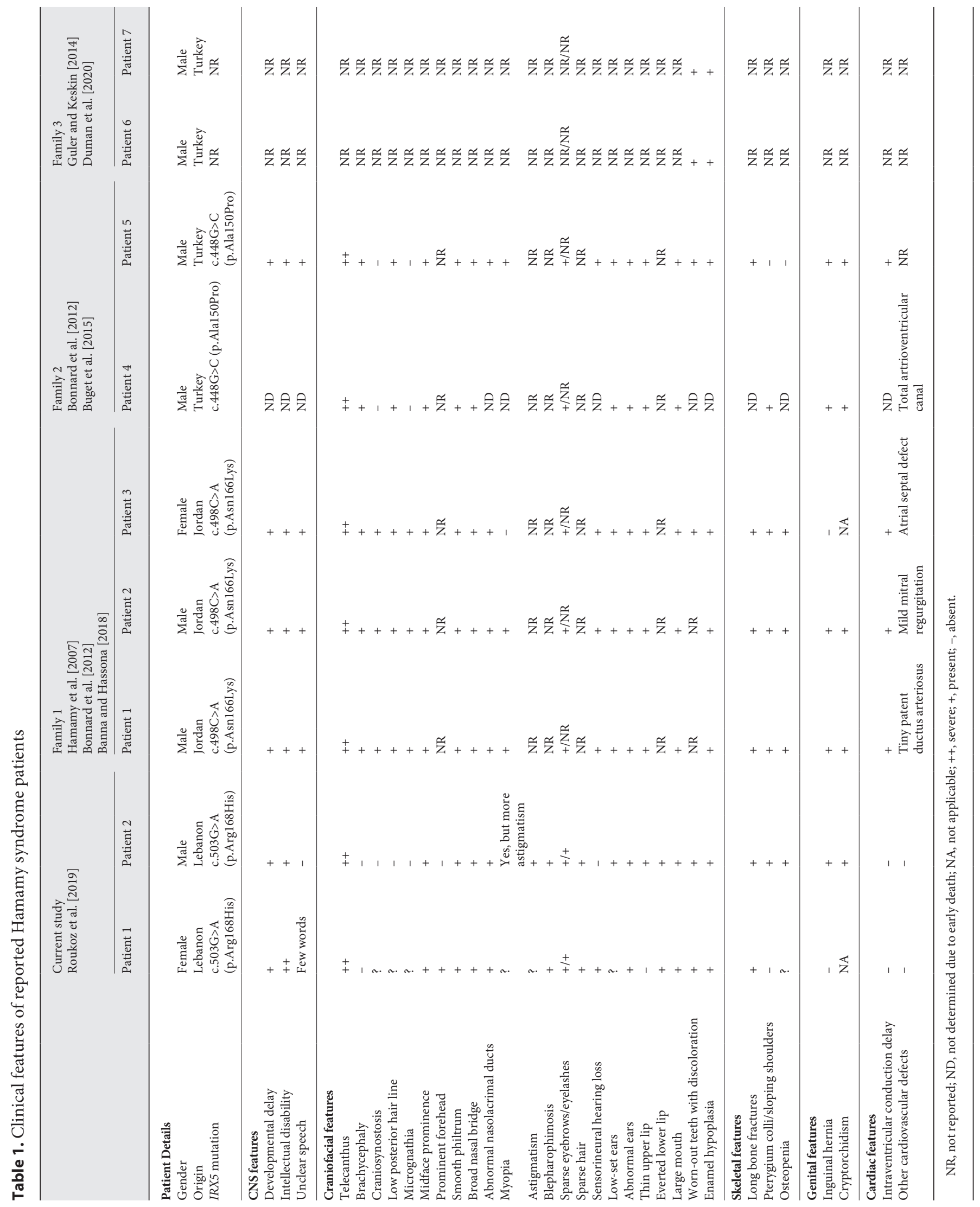


facial syndrome (608156), Opitz-GBBB syndrome (OMIM 300000), and Aarskog syndrome (OMIM 305400). In Hamamy syndrome patients, along with severe telecanthus, the overall phenotype of a prominent midface, sparse eyebrows, a broad nasal bridge, and abnormal/protruding ears results in a distinct characteristic appearance. The craniofacial features seen in our patients share striking similarities to the families reported in Hamamy et al. [2007] and Bonnard et al. [2012] (Table 1). Apart from the features mentioned above, patients from these 3 families all presented with a smooth philtrum, a large mouth, and abnormal nasolacrimal ducts.

Intellectual disability and developmental delay observed in earlier cases are also noted in our patients. The skeletal findings in the affected families also follow a similar pattern; recurrent long bone fractures, pterygium col$\mathrm{li}$, and osteopenia are seen in all reported patients. This is in line with recent findings that IRX5, the gene associated with Hamamy syndrome, plays a role in promoting osteogenesis and maintaining skeletal homeostasis, by regulating the transition of hypertrophic chondrocytes to osteoblasts [Tan et al., 2020]. Further, the genitourinary manifestations of Hamamy syndrome appear to be consistent in the reported families; all male patients exhibit inguinal hernias and cryptorchidism. Ocular anomalies are also observed in patients, although while families 1 and 2 primarily reported myopia, our patients had astigmatism as well as blepharophimosis. Interestingly, the dental findings of Hamamy patients share striking similarities, with all affected individuals reporting severe enamel hypoplasia, worn-out teeth, and grayish discoloration. This is also true for family 3 , for whom no other information has been reported [Guler and Keskin, 2014; Duman et al., 2020].

However, phenotypic differences were seen in the cardiovascular features of Hamamy patients. While families 1 and 2 reported intraventricular conduction delays, our patients did not display any cardiac anomalies. This is particularly intriguing as IRX5 has recently been identified as a regulator of human ventricular depolarization and cardiac electrical conduction [Al Sayed et al., 2021]. The lack of cardiovascular abnormalities in our patients is thus an important finding as it represents an expansion of the Hamamy phenotype, wherein affected individuals do not necessarily exhibit conduction defects.

In Hamamy patients where a molecular diagnosis was reported, mutations in $I R X 5$ were found to be the causal variants. $I R X 5$ is a 3,621-bp long gene located in the long arm of chromosome 16. It encodes a transcription factor that plays a key role in embryonic morphogenesis, particularly in craniofacial and gonadal development, by reg-

New IRX5 Mutation in Hamamy Syndrome ulating the migration of progenitor cell populations through chemokine signaling molecules [Bonnard et al., 2012]. In mice, Ir $x 5$ is responsible for establishing the cardiac ventricular repolarization gradient by repressing the $K c n d 2$ gene encoding the voltage-gated potassium channel [Costantini et al., 2005]. It has also been shown to regulate the mineralization of specific cranial bones [Cain et al., 2016] and the development of cone bipolar cells in the retina [Cheng et al., 2005].

IRX5 belongs to the Iroquois homeobox family of genes, and similar to other members of the IRX family, it consists of 2 conserved regions: a homeobox domain and an IRO box motif. The DNA-binding homeobox domain belongs to the TALE (three-amino acid-loop extension) superclass of homeodomains, which feature additional 3 amino acids between the first and second helix of 3 alpha helices [Bürglin, 1997; Cavodeassi et al., 2001]. The IRX5 mutations that have been reported thus far in Hamamy patients all fall within the helices of this domain; p.(Ala150Pro) is located in helix 2, while p.(Asn166Lys) lies in helix 3. When studied by Bonnard et al. [2012] using mouse Irx 5 expression constructs and a rat Kcnd2 promoter reporter, these 2 missense variants failed to transactivate Kcnd2. They were noted to act as hypomorphic alleles due to their altered protein stability and DNA binding ability. The p.(Arg168His) variant reported in our study also lies in helix 3 of the homeodomain, the recognition helix for specific DNA binding. Arginine 168 sits next to the p.(Asn166Lys) described by Bonnard et al. [2012] and affects a highly conserved residue (Fig. 4b, c). The Asparagine at position 166 and the Arginine at position 168 are strictly conserved across species in all homeodomains. The latter, mutated in our patients, makes direct hydrogen bonds with DNA backbones from 2 nonspecific nucleotides at the $5^{\prime}$ flanking region of the promoter recognition sequence and is evolutionarily conserved to ensure the correct positioning of the recognition helix [Chi, 2005]. Similarly to the p.(Asn166Lys) mutation, the variant reported here is thus expected to behave in a similar manner, affecting DNA binding and causing a partial loss of function of the IRX5 gene.

In conclusion, we report here a fourth family with Hamamy syndrome associated with a mutation in IRX5. Given the presence of typical Hamamy features such as developmental delay, intellectual disability, severe telecanthus, abnormal ears, dentinogenesis imperfecta, and bone fragility in the 2 patients here, and the absence of other previously described features like the cardiac phenotype, this report helps further define the spectrum of the Hamamy phenotype. 


\section{Acknowledgements}

We are thankful to the families for their collaboration.

\section{Statement of Ethics}

The research was conducted according to the ethical principles established by the World Medical Association Declaration of Helsinki. Written informed consent was obtained from the parents of both patients for the publication of this case report and the accompanying images. Informed consent for genetic analysis was obtained from the family in compliance with national ethics regulation. As so, the research did not require institutional review board, or ethical approval.

\section{Conflict of Interest Statement}

The authors have no conflicts of interest to report.

\section{Funding Sources}

This work has been supported by grants from the European Union's 7th Framework Programme for research, technological development and demonstration: LEB'IN (Lebanon-Europe "on boarding" to innovate and enhance research links in health, grant agreement 294983) and NMD-CHIP (Development of targeted DNA-Chips for High Throughput Diagnosis of NeuroMuscular Disorders, grant agreement 223026). This work was also supported by grants from the Institut Jerome Lejeune, Paris, France.

\section{Author Contributions}

A.M., S.H., and V.D. designed research. A.M., C.C., S.B., A.C., N.R.-T., D.M., and V.D. performed main experiments. C.D., J.P.D., and V.D. did the bioinformatical analysis. A.M., S.H., H.M., C.D., M.T.A.-A., S.E.-H., and V.D. wrote the paper. All authors critically analyzed, discussed and interpreted data, and edited the manuscript.

\section{References}

Adzhubei IA, Schmidt S, Peshkin L, Ramensky VE, Gerasimova A, Bork P, et al. A method and server for predicting damaging missense mutations. Nat Methods. 2010;7(4):248-9.

Al Sayed ZR, Canac R, Cimarosti B, Bonnard C, Gourraud JB, Hamamy H, et al. Human model of IRX5 mutations reveals key role for this transcription factor in ventricular conduction. Cardiovasc Res. 2021;117(9):2092-2107.

Banna SA, Hassona Y. New oral findings in Hamamy syndrome. Spec Care Dentist. 2018;38(6):445-6.

Bonnard C, Strobl AC, Shboul M, Lee H, Merriman B, Nelson SF, et al. Mutations in IRX5 impair craniofacial development and germ cell migration via SDF1. Nat Genet. 2012;44(6):709-13.

Buget MI, Canbolat N, Akgul T, Kucukay S. Anaesthesia and orphan disease: Anaesthetic management of a child with Hamamy syndrome. Eur J Anaesthesiol. 2015;32(12):891-3.

Bürglin TR. Analysis of TALE superclass homeobox genes (MEIS, PBC, KNOX, Iroquois, TGIF) reveals a novel domain conserved between plants and animals. Nucleic Acids Res. 1997;25(21): 4173-80.

Cain CJ, Gaborit N, Lwin W, Barruet E, Ho S, Bonnard $\mathrm{C}$, et al. Loss of Iroquois homeobox transcription factors 3 and 5 in osteoblasts disrupts cranial mineralization. Bone Rep. 2016;5:86-95.

Cavodeassi F, Modolell J, Gómez-Skarmeta JL. The Iroquois family of genes: from body building to neural patterning. Development. 2001;128(15): 2847-55.

Cheng CW, Chow RL, Lebel M, Sakuma R, Cheung $\mathrm{HO}$, Thanabalasingham V, et al. The Iroquois homeobox gene, Irx 5 , is required for retinal cone bipolar cell development. Dev Biol. 2005;287(1): 48-60.

Chi YI. Homeodomain revisited: a lesson from disease-causing mutations. Hum Genet. 2005; 116(6):433-44.
Costantini DL, Arruda EP, Agarwal P, Kim KH, Zhu $\mathrm{Y}, \mathrm{Zhu} \mathrm{W}$, et al. The homeodomain transcription factor Irx 5 establishes the mouse cardiac ventricular repolarization gradient. Cell. 2005; 123(2):347-58.

Desvignes JP, Bartoli M, Delague V, Krahn M, Miltgen $\mathrm{M}$, Béroud $\mathrm{C}$, et al. VarAFT: a variant annotation and filtration system for human next generation sequencing data. Nucleic Acids Res. 2018;46(W1):W545-53.

Dollfus H, Verloes A. Dysmorphology and the orbital region: a practical clinical approach. Surv Ophthalmol. 2004;49(6):547-61.

Duman SB, Dedeoglu N, Arikan B, Altun O. Sphenoid sinus agenesis and sella turcica hypoplasia: very rare cases of two brothers with Hamamy syndrome. Surg Radiol Anat. 2020;42(11):137780.

Graham A. The neural crest. Curr Biol. 2003;13(10): R381-4.

Guler C, Keskin G. Dental findings in Hamamy syndrome. Genet Couns. 2014;25(4):383-7.

Hamamy HA, Teebi AS, Oudjhane K, Shegem NN, Ajlouni KM. Severe hypertelorism, midface prominence, prominent/simple ears, severe myopia, borderline intelligence, and bone fragility in two brothers: new syndrome?. Am J Med Genet A. 2007;143A(3):229-34.

Kumar P, Henikoff S, Ng PC. Predicting the effects of coding non-synonymous variants on protein function using the SIFT algorithm. Nat Protoc. 2009;4(7):1073-81.

Li H, Durbin R. Fast and accurate short read alignment with Burrows-Wheeler transform. Bioinformatics. 2009;25(14):1754-60.

McKenna A, Hanna M, Banks E, Sivachenko A, Cibulskis K, Kernytsky A, et al. The Genome Analysis Toolkit: a MapReduce framework for analyzing next-generation DNA sequencing data. Genome Res. 2010;20(9):1297-303.
Rentzsch P, Witten D, Cooper GM, Shendure J, Kircher M. CADD: predicting the deleteriousness of variants throughout the human genome. Nucleic Acids Res. 2019;47(D1):D886-94.

Roukoz C, Kanj R, Maalouf FT, Zeinoun P. Neuropsychological Findings in Hamamy Syndrome: A Clinical Case Report. J Int Neuropsychol Soc. 2019;25(3):336-42.

Salgado D, Desvignes JP, Rai G, Blanchard A, Miltgen M, Pinard A, et al. UMD-Predictor: A HighThroughput Sequencing Compliant System for Pathogenicity Prediction of any Human cDNA Substitution. Hum Mutat. 2016;37(5):439-46.

Schwarz JM, Cooper DN, Schuelke M, Seelow D. MutationTaster2: mutation prediction for the deep-sequencing age. Nat Methods. 2014;11(4): 361-2.

Sulonen AM, Ellonen P, Almusa H, Lepistö M, Eldfors S, Hannula S, et al. Comparison of solution-based exome capture methods for next generation sequencing. Genome Biol. 2011;12(9): R94.

Tan Z, Kong M, Wen S, Tsang KY, Niu B, Hartmann C, et al. IRX3 and IRX5 Inhibit Adipogenic Differentiation of Hypertrophic Chondrocytes and Promote Osteogenesis. J Bone Miner Res. 2020; 35(12):2444-57.

Vega-Lopez GA, Cerrizuela S, Tribulo C, Aybar MJ. Neurocristopathies: New insights 150 years after the neural crest discovery. Dev Biol. 2018; 444(Suppl 1):S110-43.

Wang K, Li M, Hakonarson H. ANNOVAR: functional annotation of genetic variants from highthroughput sequencing data. Nucleic Acids Res. 2010;38(16):e164.

Watt K, Trainor PA. Chapter 17 - Neurocristopathies: the Etiology and Pathogenesis of Disorders Arising from Defects in Neural Crest Cell Development, Neural Crest Cells. Boston: Academic Press; 2014. p. 361-94 